\title{
Propiedades psicométricas de la escala de interacción en las clases dirigidas (EICD)
}

\author{
Adrián Pastor-Barceló ${ }^{1}$ D, Vicente Prado-Gascó2 ${ }^{(D)}$, Pilar Bustillo-Casero ${ }^{1}$ (D) \\ ${ }^{1}$ Departamento de Educación Física y Deportiva de la Universidad de Valencia (Spain) \\ ${ }^{2}$ Universidad Europea de Valencia (Spain)
}

pastorcs92@gmail.com, vicentejavier.prado@uem.es, pilarbustillocasero@.gmail.com

Received April, 2016

Accepted May, 2016

\section{Resumen}

Objeto: La presente investigación se centra en la construcción y validación de una escala diseñada para evaluar la calidad de la clases dirigidas: Escala de Interacción en las Clases Dirigidas (EICD).

Diseño/metodología/enfoque: Se trata de un estudio descriptivo correlacional. Para la elaboración de la escala se recurrió a tres fases diferenciadas en las que diferentes expertos evaluaron la adecuación de los ítems, finalmente se estudiaron las propiedades psicométricas de la versión final en una muestra de 314 socios (69.1\% mujeres) con edades entre los 18 y los 77 con una media de 39.33 años (DT=12.25).

Aportaciones y resultados: La escala presenta una validez y fiabilidad adecuadas, siendo una herramienta útil para la medición de la interacción en las clases dirigidas.

Limitaciones: El tipo de muestreo, no probabilístico o de conveniencia, haber tomado la muestra de una única instalación deportiva y el reducido tamaño de la muestra.

Implicaciones prácticas: La EICD permite a los gestores recibir un mejor feedback, permitiendo obtener una visión más profunda sobre la calidad y satisfacción. Acorde a los 
resultados, se podrán realizar diferentes estrategias para mejorar la calidad en un tipo de servicio clave dentro de los centros deportivos.

Originalidad / Valor añadido: Por primera vez se evalúa la interacción entre los clientes y entre clientes y empleados tanto dentro como fuera del centro, hecho que aún no se había contemplado en la literatura científica, dicha escala se podrá aplicar a cualquier tipo de actividad dirigida, y permitirá una mayor comprensión de la calidad del servicio.

Palabras clave: Calidad, Escala, Interacción, Clases dirigidas, Validación, Gestión deportiva

\section{Códigos JEL: M31}

Title: Psychometric properties of the interaction on supervised classes scale (ISCS)

\section{Abstract}

Purpose: This research focuses on the construction and validation of a scale designed to assess the quality of the supervised classes: Interaction on Supervised Classes Scale (ISCS).

Design/methodology: This is a descriptive correlational study. For the construction of the scale three phases were performed in which different experts assessed the adequacy of the items. Finally, the psychometric properties of the final version were studied in a sample of 314 consumers (69.1\% women) aged between 18 and 77 with an average of 39.33 years $(\mathrm{SD}=12.25)$.

Findings: The scale presents adequate validity and reliability, being a useful tool for measuring the interaction in Supervised Classes.

Research limitations/implications: The sampling, non-probabilistic or convenience, have taken the sample of a unique sports facility and the small sample size.

Practical implications: The ISCS allows managers to receive better feedback, allowing them to obtain deeper insight into the quality and satisfaction of the service. According to its results, the managers may implement different strategies to improve quality in a key service within sports centers.

Originality/value: For the first time the interaction between customers and between customers and employees is evaluated both inside and outside the center, a topic that had not 
yet been studied in the scientific literature. The scale can be applied to any type of directed activity, and will allow a greater understanding of the quality of service.

Keywords: Quality, Interaction, Supervised classes, Scale, Validation, Sport management

Jel Codes: M31

\section{Introducción}

El estudio de la calidad percibida en servicios deportivos se ha centrado de forma clásica en aspectos tangibles del servicio, sin embargo, los estudios más actuales se focalizan en analizar la relación de la calidad con otras variables que envuelven la gestión (Calabuig, Prado-Gascó, Crespo, Núñez-Pomar \& Añó, 2015). De entre todos los aspectos analizados, quizás la interacción entre los agentes del servicio y usuarios constituye el gran desconocido en la literatura científica. Aunque la interacción que se produce entre los clientes y, entre los clientes y empleados incide de manera clara sobre la valoración que los implicados realizan del servicio.

El primer atisbo de preocupación por este factor vino de la mano de Brady y Cronin (2001), quienes introdujeron dentro de su escala de medición de la calidad la dimensión calidad de la interacción. Esta tendencia evolucionó con Ko y Pastore (2005), al hablar de interacción cliente-cliente y clienteempleado. Posteriormente una gran cantidad de escalas han sido diseñadas para medir servicios deportivos, pero en general no han prestado atención a la interacción de forma clara o específica.

Fruto de esta laguna en el campo científico, se plantea como objetivo de esta investigación la validación de una nueva escala, llamada: Escala de Interacción en las Clases Dirigidas (EICD). El propósito de esta herramienta, es analizar la interacción entre clientes y entre clientes y empleados en diferentes espacios temporales de las clases dirigidas. Es interesante analizar la interacción en estas actividades, ya que mueven un gran volumen de gente simultáneamente, por lo que hay una mayor interacción entre clientes. Además, este tipo de servicio, constituye una pieza clave para muchas empresas deportivas.

Así, en este artículo presentamos el proceso de construcción de la EICD y el análisis de sus propiedades psicométricas. 


\section{Revisión literatura y marco teórico}

La calidad es un tema de interés desde hace años en el campo de la gestión deportiva (Calabuig, Burillo, Crespo, Mundina \& Gallardo, 2010). Pero durante muchos años, se encuentra un vacío teórico entorno al análisis de la interacción durante el servicio.

Los primeros intentos de creación de escalas de medición, fueron protagonizados por el modelo de Grönroos (1984) llamado también GM (Grönroos Model) que posteriormente fue adaptado por Rust y Oliver (1994). Con el tiempo, apareció un modelo más completo, siendo el más utilizado para la medición de la calidad del servicio en los últimos años (Kwok, Jusoh \& Khalifah, 2016; Lam, Zhang \& Jensen, 2005; Morales, Hernández-Mendo \& Blanco, 2009). Estamos hablando del modelo Servqual (Parasuraman, Zeithaml \& Berry, 1985, 1988), el cual se plasmó en una herramienta compuesta por 22 ítems y 5 categorías: Tangibles, fiabilidad, capacidad de respuesta, seguridad y empatía. Quizás la interacción se podría observar de manera implícita en la dimensión empatía y hasta cierto punto en capacidad de respuesta, si bien no fue considerada como una dimensión en el modelo.

Las críticas al modelo Servqual (Parasuraman et al., 1985, 1988) como las realizadas por Cronin y Taylor (1992), dieron lugar a la proliferación de nuevas escalas. Brady y Cronin (2001) crearon un nuevo modelo que contenía una gran novedad, fue la primera escala que incorporó el estudio de la calidad de la interacción. Se planteó un modelo donde la calidad está formada por tres dimensiones:

- Calidad de la interacción, en referencia al resultado de la interacción con el componente humano del servicio;

- Calidad del entorno, definida como los elementos tangibles del servicio;

- Calidad del resultado, que es la relación entre la calidad recibida tras el servicio y la esperada.

Cada una de estas tres dimensiones está compuesta por otras tres subdimensiones, concretamente la dimensión calidad de la interacción considera:

- Actitud. Aborda la relación con los empleados, su comportamiento educado, la disposición a ayudar al cliente y la capacidad de satisfacer las necesidades de cada uno de ellos;

- Comportamiento. Definida por el trato eficaz y rápido hacia los clientes por parte de los empleados y las ganas a la hora de trabajar; 
- Formación. Experiencia y capacidades de los empleados y su competencia cuando tienen que actuar (Brady \& Cronin, 2001).

Así, el modelo de Brady y Cronin (2001) supuso un avance en la disciplina en lo referente a la medición de la interacción dentro del servicio. Hasta entonces, los modelos anteriores no habían abordado este tema de manera explícita, únicamente se habían centrado en aspectos tangibles del servicio, dejando la interacción olvidada. La escala de Brady y Cronin (2001) ha sido utilizada en diversas áreas, incluyendo la industria del fitness (Calabuig, Quintanilla \& Mundina, 2008).

A partir del modelo de Brady y Cronin (2001) comienzan a desarrollarse nuevas escalas, aumentando el valor atribuido a la interacción dentro del servicio. Así, nace The Scale of Services Quality in Recreational Sports (SSQRS) de Ko y Pastore (2005), que tiene como objetivo identificar y evaluar los factores de la calidad del servicio dentro de la recreación deportiva. En este modelo jerárquico, vuelve a considerarse la interacción como una dimensión del servicio:

- calidad del programa,

- calidad de la interacción,

- calidad del resultado y

- calidad del ambiente.

A su vez, cada una de las dimensiones está formada por diferentes subdimensiones, en el caso de la interacción se consideran:

- Interacción cliente-empleado. Es la percepción subjetiva de la relación que se desarrolla entre el cliente y el empleado durante el servicio;

- Interacción cliente-cliente. Es la evaluación subjetiva de los clientes sobre la relación con otros usuarios en el desarrollo del servicio.

En el modelo SSQRS (Ko \& Pastore, 2005) se estudia la interacción desde una perspectiva diferente al de Brady y Cronin (2001), además de centrarse en las relaciones entre empleado y cliente, introducen una novedad en el estudio de este factor, atendiendo también a la interacción entre clientes.

Si bien estos son los modelos predominantes a nivel internacional, en España se han diseñado diferentes herramientas para la medición de la calidad percibida, como la escala NEPTUNO (Calabuig 
et al., 2008), la Eventqual (Calabuig, Mundina \& Crespo, 2010) utilizada en diferentes estudios (Crespo, Pérez-Campos \& Mundina, 2012; Mundina \& Calabuig, 2011), o la escala EPOD (Nuviala, Tamayo, Nuviala, González \& Fernández, 2010). Esta última escala contiene una dimensión denominada imagen, compuesta por 4 ítems que hablan sobre la relación con el monitor y sobre la integración del cliente en el grupo.

Concretamente en el servicio fitness, se han construido modelos para medir la calidad percibida por los usuarios. Encontramos la Services Quality Assesment Scale (SQAS) diseñada por Lam et al. (2005) y la escala CALIDFIT (García, Cepeda \& Martín, 2012). Ninguna presta especial atención a la interacción entre clientes, se centran en la relación entre clientes y los empleados y, de forma poco profunda.

Tal y como se ha expuesto, si bien la interacción constituye un aspecto básico que influirá sobre cualquier valoración del servicio, ha sido el gran olvidado en la literatura científica. Especial relevancia tiene dicho factor sobre las denominadas clases dirigidas, uno de los ejes centrales de la actividad de cualquier servicio deportivo (Calabuig, Núñez-Pomar, Prado-Gascó \& Añó, 2014).

Por todo ello, el objetivo de la presente investigación ha sido desarrollar y validar un instrumento para evaluar la interacción que se produce entre el cliente y empleado y entre clientes en las clases dirigidas, que recibe el nombre de "Escala de Interacción en las Clases Dirigidas (EICD)", compuesta por tres dimensiones que responden a tres espacios temporales distintos de las clases: antes (pre), durante (durante) y después (post).

\section{Metodología}

\subsection{Participantes}

Los participantes del estudio fueron 314 socios de una instalación fitness premium de una localidad del área metropolitana de Valencia, que tiene un total de 1554 socios. De los 314 socios 97 son hombres $(30.9 \%)$ y 217 mujeres (69.1\%). La edad oscila entre los 18 y los 77 con una media de 39.33 años $(\mathrm{DT}=12.25)$. 


\subsection{Procedimiento de creación del instrumento}

Para la creación de la EICD se desarrollaron tres fases diferenciales:

- $1^{\mathrm{a}}$ Fase. En primer lugar partiendo de las 3 dimensiones que componen el modelo teórico de partida, dos tipos de interacción y tres momentos de interacción en las clases, se desarrolló un pull de 23 ítems: 9 para antes (pre), 6 para durante y 8 para después (post). Posteriormente, se presentaron dichos ítems a un grupo de 3 expertos, los cuales debían indicar la adecuación e importancia de cada uno de los ítems para cada una de las dimensiones objeto de estudio. En base a las respuestas de los expertos algunos ítems fueron reformulados y otros eliminados, dando lugar a 5 ítems en la dimensión pre, 4 en durante y 5 en post.

- $\quad 2^{a}$ Fase. A continuación, una vez realizada la primera depuración de la escala, se presentó la versión preliminar del instrumento a una nueva comisión de 2 expertos. A partir de las respuestas dadas por los expertos se estableció el término compañeros/as de clase en los ítems de interacción entre clientes. Además, se unificaron criterios en los ítems que respondían al mismo espacio temporal.

- $\quad 3^{a}$ Fase. Seguidamente se procedió con una tercera fase en la que la escala fue revisada por otra comisión de expertos. Se hizo una depuración de los ítems que trajo cambios importantes: se añadió un rasgo de frecuencia a través de la palabra suelo, se cambiaron expresiones como interactuar y rodear por la palabra hablar y, finalmente, se introdujeron dos ítems en relación a las redes sociales en la dimensión post. La escala resultante de esta tercera fase fue la que se pasó a los 314 participantes de la investigación y cuyas propiedades psicométricas se analizan en la presente investigación.

Así, la escala final queda compuesta por 16 ítems divididos en 3 dimensiones. La dimensión pre consta de 5 ítems, de los cuales 4 hacen referencia a la interacción cliente-cliente y 1 a cliente-empleado. En la dimensión durante encontramos 4 ítems, 3 ítems sobre la interacción cliente-cliente y 1 ítem sobre cliente-empleado. Por último, la dimensión post tiene 7 ítems, siendo 4 de ellos de interacción clientecliente y 3 de cliente-empleado. 


\subsection{Instrumentos}

Para la recogida de la información se recurrió a un instrumento autoadministrable compuesto por la escala EICD y por una serie de preguntas sociodemográficas (edad, sexo, ...).

\subsection{Procedimiento}

Los datos fueron obtenidos a partir de los usuarios de una instalación fitness Premium. El tiempo de contestación a la encuesta fue de 5 minutos aproximadamente, la respuesta a la misma se hizo de forma anónima y voluntaria. Los cuestionarios fueron administrados en dos puntos diferentes: Antes de las clases esperando a entrar en las salas y en la entrada de los vestuarios.

\subsection{Análisis de datos}

El análisis estadístico de los datos, fue realizado mediante los paquetes estadísticos SPSS 20 y EQS 6.2. Para el estudio de los datos se llevaron a cabo diferentes procedimientos estadísticos.

En primer lugar, se calcularon los estadísticos descriptivos de los ítems de la escala. Posteriormente, se efectuó un análisis de fiabilidad, calculando el alfa de Cronbach, no obstante, ya que este índice no tiene en cuenta la influencia del resto de constructos sobre la fiabilidad, se calcularon la varianza media extraída (AVE) y la fiabilidad compuesta (FC). Para el estudio de la validez del instrumento, se analizaron la validez factorial y discriminante.

\section{Resultados}

\subsection{Análisis de los ítems y fiabilidad}

Los 16 ítems que componen la escala fueron sometidos a diversos AFE y AFC, la versión resultante que obtiene adecuados índices de ajuste es la compuesta por 11 ítems, cuyas propiedades se presentan a continuación. En la Tabla 1 se presenta la composición final de los ítems junto con los siguientes datos para cada uno de ellos: media $(\mathrm{M})$, desviación típica (DT), correlación ítem-total $\left(\mathrm{r}_{\mathrm{j} x}\right)$ y el alfa de Cronbach si se elimina el elemento $(\alpha-\mathrm{x})$. 


\begin{tabular}{|c|c|c|c|c|}
\hline Ítem & $\mathbf{M}$ & DT & $\mathbf{r}_{\mathrm{jx}}$ & $\alpha-x$ \\
\hline \multicolumn{5}{|c|}{$\begin{array}{l}\text { Dimensión 1: Interacción dentro del centro con compañeros de clase } \alpha: .89 \text {; AVE:.65; } \\
\text { FC:.90 }\end{array}$} \\
\hline $\begin{array}{l}\text { Conozco a la mayor parte de mis } \\
\text { compañeros de clase }\end{array}$ & 2.96 & 1.21 & .72 & .87 \\
\hline $\begin{array}{l}\text { Antes de la clase suelo hablar con los } \\
\text { demás compañeros de clase }\end{array}$ & 3.11 & 1.19 & .68 & .88 \\
\hline $\begin{array}{l}\text { Tengo una buena relación con la mayor } \\
\text { parte de mis compañeros de clase }\end{array}$ & 3.26 & 1.17 & .65 & .88 \\
\hline $\begin{array}{l}\text { Al acabar la clase suelo hablar con mis } \\
\text { compañeros }\end{array}$ & 3.15 & 1.18 & .67 & .88 \\
\hline $\begin{array}{l}\text { Suelo acudir a las clases dirigidas para ver a } \\
\text { mis compañeros de clase }\end{array}$ & 2.71 & 1.24 & .61 & .88 \\
\hline \multicolumn{5}{|c|}{$\begin{array}{l}\text { Dimensión 2: Interacción dentro del centro con empleados y otros clientes } \alpha: .86 \text {; } \\
\text { AVE:.68; FC:.86 }\end{array}$} \\
\hline $\begin{array}{l}\text { Durante la clase suelo hablar con otras } \\
\text { personas aunque no las conozca }\end{array}$ & 2.67 & 1.15 & .53 & .88 \\
\hline $\begin{array}{l}\text { Durante la clase me gusta hablar cada vez } \\
\text { con personas diferentes }\end{array}$ & 2.68 & 1.18 & .59 & .88 \\
\hline $\begin{array}{l}\text { Durante la clase suelo hablar con el } \\
\text { monitor }\end{array}$ & 2.53 & 1.20 & .59 & .88 \\
\hline \multicolumn{5}{|c|}{$\begin{array}{l}\text { Dimensión 3: Interacción fuera del centro con clientes y empleados } \alpha: .85 \text {; AVE:.67; } \\
\text { FC: } 86\end{array}$} \\
\hline $\begin{array}{l}\text { Sigo a alguno de los monitores de clase a } \\
\text { través de las redes sociales }\end{array}$ & 1.98 & 1.31 & .52 & .89 \\
\hline $\begin{array}{l}\text { Sigo a parte de mis compañeros de clase a } \\
\text { través de las redes sociales }\end{array}$ & 2.01 & 1.33 & .62 & .88 \\
\hline $\begin{array}{l}\text { A veces quedo con el monitor fuera de la } \\
\text { instalación }\end{array}$ & 1.74 & 1.14 & .54 & .88 \\
\hline
\end{tabular}

Tabla 1. Análisis de los ítems y fiabilidad de la escala

En general, los resultados muestran que la EICD tiene en conjunto una fiabilidad adecuada $(\alpha=.89)$ y que todos los ítems parecen contribuir de forma adecuada al instrumento. Presentan una correlación relativamente alta con el conjunto de la escala y no se observa que mejore el instrumento al eliminar alguno de ellos, ya que $\alpha$-x se sitúa entre .87 y .89 según el ítem.

Considerando las diferentes dimensiones, estas también parecen adecuadas, presentando valores entre . 85 y .89 (dimensión $1 \alpha=.89$; dimensión $2 \alpha=.86$ y dimensión $3 \alpha=.85$ ). La consistencia interna se estudió utilizando el alfa de Cronbach, no obstante este indicador no tiene en cuenta la influencia de otros constructos, por lo que se llevó a cabo el análisis de la varianza media extraída (AVE) (Fornell \& Larcker, 1981) y de la fiabilidad compuesta (FC). El AVE para considerarse un valor adecuado tiene que superior a .50 (Bagozzi \& Yi, 1988) y para los valores de la fiabilidad compuesta se recomiendan que sean superiores a .70 (Nunnally, 1978). Los valores de FC y AVE de cada dimensión son aceptables siendo superiores a los recomendados (ver Tabla 1). 


\subsection{Análisis de validez}

La validez factorial y la validez discriminante fueron estudiadas. La validez de contenido no fue nuevamente analizada, ya que ésta estuvo presente en el propio desarrollo del instrumento, al someter la escala a juicio de experto en tres ocasiones diferentes. En primer lugar, fue evaluada la adecuación de la muestra para la realización de AFE mediante la prueba de Kaiser-Meyer-Olkin $(\mathrm{KMO}=.87)$ y la prueba de esfericidad de Barlett $(\mathrm{p}<.01)$, confirmando la adecuación de los datos. Se procedió a realizar el AFE, utilizando el método de componentes principales con rotación Varimax y criterios de extracción autovalores iguales o mayores a 1. Se observó una estructura formada por tres factores, explicando un $70.95 \%$ de la varianza total. El primer factor explicó el $47.06 \%$ de la varianza, el segundo el $11.92 \%$ y el tercer factor el 11.43\%. 3 ítems de la escala final fueron eliminados debido a tener cargas factoriales menores a .40, con lo que la escala quedó compuesta tras el AFE por un total de 13 ítems.

El primer factor, fue llamado Interacción dentro del centro con compañeros de clase, los ítems dentro de este factor, hacen referencia a la interacción dentro de la instalación con usuarios que el cliente ya conoce. El segundo factor, está compuesto por ítems sobre la interacción que se produce dentro de la instalación con empleados y usuarios desconocidos para el cliente, fue llamado Interacción dentro del centro con empleados y otros clientes. Por último, los ítems que saturaron en el tercer factor estaban relacionados con la interacción cliente-cliente y cliente-empleado fuera de la instalación. Este tercer factor fue llamado Interacción fuera del centro con clientes y empleados. Por lo tanto, tras el AFE la escala quedó definida por un nuevo criterio, la interacción cliente-cliente y cliente-empleado dentro y fuera de la instalación. En la Tabla 2 se observa el autovalor y la varianza explicada de cada dimensión, junto a la saturación de cada ítem. 


\begin{tabular}{|c|c|}
\hline Ítems & Carga factorial \\
\hline \multicolumn{2}{|c|}{$\begin{array}{l}\text { Dimensión 1: Interacción dentro del centro con compañeros de clase. Autovalor }=6.19 ; \% \text { de la } \\
\text { varianza }=47.60\end{array}$} \\
\hline Conozco a la mayor parte de mis compañeros de clase & .871 \\
\hline Antes de la clase suelo hablar con los demás compañeros de clase & .864 \\
\hline Tengo una buena relación con la mayor parte de mis compañeros de clase & .857 \\
\hline $\mathrm{Al}$ acabar la clase suelo hablar con mis compañeros & .736 \\
\hline Suelo acudir a las clases dirigidas para ver a mis compañeros de clase & .615 \\
\hline \multicolumn{2}{|c|}{$\begin{array}{l}\text { Dimensión 2: Interacción dentro del centro con empleados y otros clientes. Autovalor }=1.55 ; \% \text { de la } \\
\text { varianza }=11.92\end{array}$} \\
\hline Durante la clase suelo hablar con otras personas aunque no las conozca & .880 \\
\hline Durante la clase me gusta hablar cada vez con personas diferentes & .856 \\
\hline Durante la clase suelo hablar con el monitor & .764 \\
\hline $\mathrm{Al}$ acabar la clase suelo hablar con el monitor & .509 \\
\hline \multicolumn{2}{|c|}{$\begin{array}{l}\text { Dimensión 3: Interacción fuera del centro con clientes y empleados. Autovalor }=1.49 ; \% \text { de la } \\
\text { varianza }=11.43\end{array}$} \\
\hline Sigo a alguno de los monitores de clase a través de las redes sociales & .869 \\
\hline Sigo a parte de mis compañeros de clase a través de las redes sociales & .838 \\
\hline A veces quedo con el monitor fuera de la instalación & .805 \\
\hline A veces vuelvo a casa con compañeros de clase & .528 \\
\hline
\end{tabular}

Tabla 2. Matriz componentes rotados

Con el objetivo de profundizar en el estudio de la validez del instrumento a continuación se procedió a desarrollar un análisis factorial confirmatorio (AFC), eliminándose dos ítems por tener una carga factorial baja, uno de la dimensión 2 y otro de la 3, estableciéndose una escala final compuesta por 11 ítems.

Se efectuó el análisis de la validez factorial del instrumento con el método de la corrección robusta de Satorra-Bentler (Bentler, 1995), ya que el coeficiente de Mardia (Mardia, 1970) presentó un resultado de 43.05, siendo superior a 5, lo que aconseja utilizar este método de análisis. Fueron analizados indicadores como el ratio entre $\chi^{2}$ y sus grados de libertad y el ratio entre S-B $\chi^{2}$ y sus grados de libertad, siendo un dato adecuado valores menores de 5 (Byrne, 1989). También se presentan en la Tabla 3 otros indicadores robustos que fueron estudiados como el normed fit index (NFI), el nonnormed fit index (NNFI) y el comparative fit index (CFI). Estos datos fueron adecuados, ya que son aceptables valores por encima de .90 (Maccallum \& Austin, 2000). Otro de los indicadores estudiados fue el root mean-square error of approximation (RMSEA) robusto, mostrando su adecuación a los valores recomendados por debajo de .08 (Browne \& Cudeck, 1993).

\begin{tabular}{|c|c|c|c|c|c|}
\hline$\chi^{\mathbf{2}} \mathbf{( g l )}$ & $\mathbf{S - B} \boldsymbol{\chi}^{\mathbf{2}} \mathbf{( g \mathbf { l } )}$ & NFI & NNFI & CFI & RMSEA \\
\hline $122.677(41)$ & $94.7914(41)$ & .94 & .96 & .97 & .067 \\
\hline
\end{tabular}

Tabla 3. Índices de bondad de ajuste de la EICD 
Por último, se evaluó la validez discriminante mediante el test de la varianza media extraída (AVE) (Fornell \& Larcker, 1981). Para determinar que existe validez discriminante, la raíz cuadrada de AVE tiene que ser mayor que las correlaciones entre las dimensiones o factores considerados. Los resultados que se exponen en la Tabla 4 evidencian la existencia de validez discriminante.

\begin{tabular}{|l|r|r|r|}
\cline { 2 - 4 } \multicolumn{1}{c|}{} & Dimensión 1 & Dimensión 2 & Dimensión 3 \\
\hline Dimensión 1 & $\mathbf{( . 8 1 )}$ & & \\
\hline Dimensión 2 & .49 & $\mathbf{( . 8 2 )}$ & $\mathbf{( . 8 1 )}$ \\
\hline Dimensión 3 & .46 & .37 & * \\
*En la diagonal se encuentra la raíz de AVE de cada dimensión ** Todas las \\
correlaciones son significativas a $\mathrm{p}<.01$
\end{tabular}

Tabla 4. Validez discriminante

\subsection{Correlaciones entre las dimensiones}

Las tres dimensiones que componen la escala están correlacionadas de manera moderada positiva y significativa $(\mathrm{p} \leq .01)$. La correlación más alta se observa entre la dimensión 1 y 2, y entre la 2 y la 3, y la más baja, entre la dimensión 2 y 3.

\section{Conclusiones}

Por lo que se ha podido constatar tras la revisión bibliográfica, la interacción en el servicio no ha sido un tema estudiado en profundidad. No se contempló el análisis de este factor hasta la aparición de la escala de Brady y Cronin (2001) que introdujo la evaluación de la calidad de la interacción. Con Ko y Pastore (2005) se dio un paso más, analizando tanto la interacción cliente-cliente como clienteempleado, pero todo ello no caló en la literatura, ya que en instrumentos posteriores únicamente se volvió a evaluar la relación entre clientes y empleados.

Por todo ello, se ha planteado como objetivo de esta investigación, la creación y posterior validación de una escala para evaluar la interacción que se produce en las clases dirigidas, recibiendo el nombre de Escala de Interacción en las Clases Dirigidas (EICD). Ésta, se compone de tres dimensiones diferenciales: interacción entre clientes que tienen una relación, entre clientes que no se conocen y entre los clientes y empleados, tanto fuera como dentro de la instalación. Los resultados obtenidos sugieren adecuadas propiedades psicométricas del instrumento, lo que justifica su utilización. 
Las clases dirigidas, tal y como se ha expuesto, suponen una parte importante de la actividad de cualquier instalación deportiva. Los gestores necesitan cada vez más, poder contar con información útil y veraz que le pueda guiar en sus decisiones, por ello, la creación y validación de herramientas como la EICD son de gran importancia en el sector, al permitir una toma de decisiones más eficaz.

Si bien la presente investigación cuenta con ciertas limitaciones, como el reducido tamaño de la muestra, la consideración de una única instalación, y recurrir a muestreo incidental o de conveniencia. No obstante, consideramos que supone un primer acercamiento muy útil para la disciplina, de un área tradicionalmente obviada y clave dentro de la actividad deportiva. Futuras investigaciones con la EICD deberían ampliar la muestra, considerar otros contextos o actividades fuera del fitness, así como analizar la relación que tiene la EICD con otras variables como la satisfacción o las intenciones futuras y con variables de marca como la confianza en la marca o la imagen corporativa. Otra línea que podría seguirse es el estudio de los antecedentes de la escala EICD, conociendo está información se podrían desarrollar diferentes estrategias por parte del gestor para mejorar este factor y consecuentemente aumentaría la calidad percibida del servicio.

\section{Referencias}

Bagozzi, R., \& Yi, Y. (1988). On the evaluation of structural equation models. Journal of the Academy of Marketing Science, 16, 74-94. http://dx.doi.org/10.1007/BF02723327

Bentler, P. (1995). EQS structural equations program manual. Encino, CA: Multivariate Software.

Brady, M.K., \& Cronin, J.J. (2001). Some new thoughts on conceptualizing perceived service quality: A hierarchical approach. Journal of Marketing, 65, 34-49. http://dx.doi.org/10.1509/jmkg.65.3.34.18334

Browne, M., \& Cudeck, R. (1993). Alternative ways of assessing model fit. En K. Bolleny J. Long (Eds.), Testing Structural Equation Models (pp. 136-162). Beverly Hills, CA: Sage.

Byrne, B. (1989). A primer of LISREL: Basic applications and programming for confirmatory factor analytic models. New York: Springer-Verlag. http://dx.doi.org/10.1007/978-1-4613-8885-2

Calabuig, F., Burillo, P., Crespo, J., Mundina, J.J., \& Gallardo, L. (2010). Satisfacción, calidad y valor percibido en espectadores de atletismo. Revista Internacional de Medicina y Ciencias de la Actividad Física y el Deporte, 10(40), 577-593. 
Calabuig, F., Mundina, J., \& Crespo, J. (2010). Eventqual: Una medida de calidad percibida por los espectadores de eventos deportivos. Retos. Nuevas tendencias en Educación Física, Deporte y Recreación, 18, 66-70.

Calabuig, F., Núñez-Pomar, J., Prado-Gascó, V., \& Añó, V. (2014). Effect of price increases on future intentions of sport consumers. Journal of Business Research, 67(5), 729-733. http://dx.doi.org/10.1016/j.jbusres.2013.11.035

Calabuig, F., Prado-Gascó, V., Crespo, J., Núñez-Pomar, J., \& Añó, V. (2015). Spectator emotions: Effects on quality, satisfaction, value, and future intentions. Journal of Business Research, 68(7), 1445-1449. http://dx.doi.org/10.1016/j.jbusres.2015.01.031

Calabuig, F., Quintanilla, I., \& Mundina, J. (2008) La calidad percibida de los servicios deportivos: diferencias según instalación, género, edad y tipo de usuario en servicios náuticos. Revista Internacional de Ciencias del Deporte, 10(4), 25-43. http://dx.doi.org/10.5232/ricyde2008.01003

Crespo, J., Pérez-Campos, C., \& Mundina, J. (2012). Calidad de servicio percibida por los espectadores de futbol. Análisis de diferencias entre grupos. Journal of Sports Economics \& Management, 2(1), 4-15.

Cronin, J.J., \& Taylor, S.A. (1992). Measuring Service Quality: A re-examination and extension. Journal of Marketing, 56(3), 55-68. http://dx.doi.org/10.2307/1252296

Fornell, C., \& Larcker, D. (1981). Evaluating structural equations models with unobservable variables and measurement error. Journal of Marketing Research, 18, 39-50. http://dx.doi.org/10.2307/3151312

García, J., Cepeda, G., \& Martín, D. (2012). La satisfacción de clientes y su relación con la percepción de calidad en Centro de Fitness: utilización de la escala CALIDFIT. Revista de Psicología del Deporte, 21(2), 309-319.

Grönroos, C. (1984). A Service Quality Model and its Marketing Implications. European Journal of Marketing, 18(4), 36-44. http://dx.doi.org/10.1108/EUM0000000004784

Ko, Y.J., \& Pastore, D.L. (2005). A Hierarchical Model of Service Quality for the Recreational Sport Industry. Sport Marketing Quaterly, 14(2), 84-97.

Kwok, S.Y., Jusoh, A., \& Khalifah, Z. (2016). The influence of Service Quality on Satisfaction: Does gender really matter?. Intangible capital, 12(2), 444-461. http://dx.doi.org/10.3926/ic.673

Lam, E., Zhang, J., \& Jensen, B. (2005). Service Quality Assessment Scale (SQAS): An Instrument for Evaluating Service Quality of Health-Fitness Clubs. Measurement in Physical Education and Exercise Science, 9(2), 79-111. http://dx.doi.org/10.1207/s15327841mpee0902_2 
Maccallum, R., \& Austin, J. (2000). Applications of structural equation modeling in psychological research. Annual Review of Psychology, 51, 201-226. http://dx.doi.org/10.1146/annurev.psych.51.1.201

Mardia, K.V. (1970). Measures of multivariate skewness and kurtosis with applications. Biometrika, 57(3), 519-530. http://dx.doi.org/10.1093/biomet/57.3.519

Morales, V., Hernández-Mendo, A., \& Blanco, A. (2009). Evaluación de la calidad en organizaciones deportivas: Adaptación del modelo Servqual. Revista de Psicología del Deporte, 18(2), 137-150.

Mundina, J., \& Calabuig, F. (2011). La Calidad de servicio percibida de un gran evento deportivo como inndicador de gestión. Journal of Sports Economics \& Management, 1(1), 31-47.

Nunnally, J. (1978). Psychometric theory. New York: McGraw-Hill.

Nuviala, A., Tamayo, J.A., Nuviala, R., González, J.A., \& Fernández, A. (2010). Propiedades psicométricas de la escala de valoración de organizacines deportivas EPOD. Retos. Nuevas tendencias en Educación Física. Deporte y Recreación, 18, 82-87.

Parasuraman, A., Zeithaml, V.A., \& Berry, L.L. (1985). A Conceptual Model of Service Quality and its Implications for Future Research. Journal of Marketing, 49, 41-50. http://dx.doi.org/10.1093/biomet/57.3.519

Parasuraman, A., Zeithaml, V.A., \& Berry, L.L. (1988). SERVQUAL: A Multiple-Item Scale for Measuring Consumer Perceptions of Service Quality. Journal of Retailing, 64(1), 12-40.

Rust, R.T., \& Oliver, R.L. (1994). Service quality: Insights and managerial implications from the frontier. In R.T. Rust \& R.L. Oliver (Eds.), Service quality: New directions in theory and practice (pp. 1-19). London: Sage. http://dx.doi.org/10.4135/9781452229102.n1

Intangible Capital, 2016 (www.intangiblecapital.org)

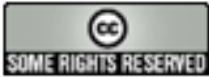

Article's contents are provided on an Attribution-Non Commercial 3.0 Creative commons license. Readers are allowed to copy, distribute and communicate article's contents, provided the author's and Intangible Capital's names are included. It must not be used for commercial purposes. To see the complete license contents, please visit http://creativecommons.org/licenses/by-nc/3.0/. 